\title{
Cosserat-Cartan modification of Einstein-Riemann relativity and cosmic dark energy density
}

\author{
Mohamed S. EI Naschie \\ Dept. of Physics, Faculty of Science, University of Alexandria, Alexandria, Egypt
}

Email address:

Chaossf@aol.com

To cite this article:

Mohamed S. El Naschie. Cosserat-Cartan Modification of Einstein-Riemann Relativity and Cosmic Dark Energy Density. American Journal of Modern Physics. Vol. 3, No. 2, 2014, pp. 82-87. doi: 10.11648/j.ajmp.20140302.17

\begin{abstract}
Based on pioneering works by Sciama and Kibble to extend Einstein-Cartan theory of gravity we give a new derivation for the cosmic energy density. It is argued that the 't Hooft-Veltman and Wilson method of renormalization implies the relativity of fractal spacetime at the quantum scale and a dark energy density of $E(D)=95.5$ percent. It is further revealed that similar conclusions could be made using A.C. Eringen's nonlocal elasticity. Finally the wider philosophical implication of the theory is discussed.
\end{abstract}

Keywords: Kibble Gravity, 't Hooft Fractal Spacetime, Dimensional Regularization, Wilson Renormalization, Cosmic Dark Energy, Sciama-Kibble gravity, Cantorian Spacetime, nonlocal elasticity,

Cantorian philosophy of science

\section{Introduction}

The present letter gives a novel and revealing derivation of a relatively new but quite well established cosmic dark energy density $E(D)=m c^{2}(21 / 22)$. The new analysis goes far beyond being an additional confirmation of this formula which is quantitatively in excellent agreement with all cosmological measurements [1-12]. We start by returning to some fundamental insights due to the work of T.W. Kibble in England [13], F.W. Hehl in Germany [14] and M. Blagojevic in Serbia who in various ways deepened our understanding of the pioneering work of the brothers Cosserat in France and the subsequent reformulation of Einstein-Riemann curvature based relativity theory by E. Cartan as well as Einstein himself [15-19]. The new idea centers around a torsion tensor which exists naturally in engineering theory of elasticity in addition to coupled stresses reminiscent of quantum spin $[17,19]$.

\section{Background Information}

The reader familiar with the geometrically and topologically intrinsic quantization of Cantorian-fractal spacetime knows that these mathematical extremely demanding modifications of general relativity fall into place effortlessly in Cantorian formulation using the golden mean binary number system or if you want the hardwareless golden mean computer $[20,21]$. This miraculous number system guides the calculation and we obtain an almost exact solution even when we set at the end the transfinite part equal zero [4-12]. In a sense the method is like the Kibble mechanism as well as ' $t$ Hooft-Veltman renormalization where we use $D-\epsilon$ then let $\in \rightarrow 0[22,23]$. Similarly $k=2 \phi^{5}=\phi^{3}\left(1-\phi^{3}\right)$ $=0.18033989$ is just like a scaffolding without which we could not erect the building but must be taken down at the end of the construction having done what was needed to be done [22-31]. In our case for instance the exact expression for the dark energy density [4-12]

$$
\mathrm{E}(\mathrm{D})=\left(5 \phi^{2} / 2\right) \mathrm{mc}^{2}
$$

where $\phi^{2}$ is the Hausdorff dimension of an empty Cantor set $\mathrm{D}(-1)=\left(-1, \phi^{2}\right) \quad([6-8]$ and $\phi=2 /(\sqrt{5}+1)$ could be written as

$$
\begin{aligned}
\mathrm{E}(\mathrm{D}) & =\left(\frac{21+\mathrm{k}}{22+\mathrm{k}}\right) \mathrm{mc}^{2} \\
= & \frac{21.18033989}{22.18033989} \mathrm{mc}^{2} \\
& \simeq\left(\mathrm{mc}^{2}\right)(21 / 22),
\end{aligned}
$$

where $22=26-\mathrm{D}^{(4)}, 21=26-\mathrm{D}^{(5)}, 26$ are the Bosonic 
string dimensions, $\mathrm{D}^{(4)}=4, \mathrm{D}^{(5)}=5$ are the dimensions of Einstein's space-time and Kaluza-Klein spacetime respectively.

However ignoring from the beginning $\mathrm{k}=\phi^{3}\left(1-\phi^{3}\right)=$ 0.18033989 compared to $\mathrm{D}=4$ would have led us to the wrong result or more accurately a result which does not distinguish between ordinary energy density $\mathrm{E}(\mathrm{O})=\mathrm{mc}^{2} / 22$ and the dark energy density $\mathrm{E}(\mathrm{D})=\mathrm{mc}^{2}(21 / 22)$ such as the classical formula of Einstein $E=m^{2}$ [20-27]. The present analysis is due to space limitation necessarily brief and presupposes reasonable familiarity with the Cosserat and Cartan modification of general relativity $[15,16]$ as well as the two related but distinct roles of torsion and micropolar stresses $[14,17]$.

\section{Analysis}

We begin our short analysis by noting that in three dimensions there is no difference in the number of independent components of $\mathrm{R}^{\alpha \beta}, \mathrm{T}^{\alpha}, \Gamma^{\alpha \beta}$ nor in fact $\vartheta^{\alpha}$ so that in all the four cases we have 9 components [14-20]. The vital difference exists however for the crucial four dimensionality where we have for the above mentioned four quantities the values $36,24,24$ and 16 respectively. Following the standard abbreviated notation by writing dim four dimensions and deg for degrees of freedom, we obtain the following scaling-like vital ratio for the four dimensional Einstein spacetime

$$
\begin{aligned}
\gamma(\mathrm{O}) & =\operatorname{deg} \mathrm{T}^{\alpha} / \operatorname{dim} \mathrm{K}_{\mathrm{v}}^{(32)} \\
& =\left|\mathrm{T}^{\alpha}\right| /\left|\mathrm{K}_{\mathrm{v}}^{(32)}\right| \\
& =24 / 528 \\
& =1 / 22
\end{aligned}
$$

where $\left|\mathrm{K}_{\mathrm{v}}{ }^{(32)}\right|$ is the number of killing vector fields for $\mathrm{n}=32$ which is equal to the number of quantum states in Witten's five Brane model in the $\mathrm{D}=11$ dimensions of M-theory $[23,24]$. This result could be written in the more familiar form discussed previously as

$$
\begin{aligned}
\gamma(\mathrm{O}) & \left.=\mathrm{N}_{\mathrm{k}}^{\left({ }^{(32)}\right.}-8(\mathrm{~N}(\mathrm{SM}))\right] / \mathrm{N}_{\mathrm{k}}^{(32)} \\
& =\frac{528-8(63)}{528}=\frac{528-504}{528} \\
& =\frac{1}{22}
\end{aligned}
$$

where 504 is the well known number of states of Heterotic strings [23,32]. However in the above form we could give $\gamma_{o}$ a radical totally unorthodox interpretation or at least an analogy as a high energy quasi Doppler effective scaling. In other words 528 plays the role of he wave length of emitted photons while 504 plays the role of the wave length of absorber photons. This applies of course to ordinary energy scaling of Einstein's maximal energy density and leads to $\mathrm{E}(\mathrm{O})=\gamma(\mathrm{O}) \mathrm{mc}^{2}=\mathrm{mc}^{2} / 22$. For dark energy on the other hand we see that a negative sign appears for $\gamma(D)$ which is given by the obvious expression

$$
\gamma(\mathrm{D})=\frac{-504}{528}=\frac{-21}{22}
$$

leading to a second by now also familiar density namely the cosmic dark energy density

$$
\begin{aligned}
\mathrm{E}(\mathrm{D}) & =\left|\gamma(\mathrm{D}) \mathrm{mc}^{2}\right| \\
& =\left|-(21 / 22) \mathrm{mc}^{2}\right| \\
& =m c^{2}(21 / 22) .
\end{aligned}
$$

Interpreting $4-\epsilon$ as a real fractal dimension as we do here is definitely a new idea in high energy physics but it is by no means new in solid state physics [24-27]. For instance Ising gauge theory in the famous 3.9999 dimensions of the late Nobel Laureate and extraordinary wizard of computerized calculation, K. Wilson [29-31] was investigated in the eighties of last century in gauge theory and critical phenomena by many authors. However the fractal interpretation was not taken literally to mean that spacetime is really a fractal. In our case by contrast we are persuaded and hope to persuade that both the theoretical results and the cosmological measurements that $\gamma=(4-\mathrm{k}) / 4 \simeq 0.95$ means that between $10^{16} \mathrm{Gev}$ and $10^{19} \mathrm{Gev}$ spacetime possesses a fractal Hausdorff dimension equal $4-\mathrm{k}$ and that by T-duality the same applies at the "edge" of the hyperbolic fractal holographic universe. In fact the present Author made some numerical estimations of the dimensionality of spacetime long before discovering the intimate relation between dark energy density and 't Hooft's dimensional regularization $[22,23]$. This result found almost 17 years ago was a volume based dimension [31]:

$$
\begin{aligned}
\mathrm{D} & \simeq\left\langle\mathrm{V}\left(\mathrm{S}^{(\infty)}\right)\right\rangle \\
& =\sum_{0}^{\mathrm{n}=24} \mathrm{nV}\left(\mathrm{S}^{(\mathrm{n})}\right) \Delta_{\mathrm{n}} / \sum_{0}^{24} \mathrm{n} \Delta_{\mathrm{n}} \\
& \simeq 3.8
\end{aligned}
$$

whereas the exact result of our present exact theory is $\mathrm{D}=4$ $-\mathrm{k}=3.819660110$ [28]. This last exact value could be rewritten as

$$
\begin{aligned}
\mathrm{D} & =(10)\left(\phi^{2}\right) \\
& =(10)(0.3819660110) .
\end{aligned}
$$

Since $\phi^{2}$ is the Hausdorff dimension of the empty set, D in this case can be seen as D $=4$ minus ten copies of the empty set or alternatively 4 minus twice the five dimensional volume of Kaluza-Klein's empty set, i.e. $2\left(5 \phi^{5}\right)$ where $\phi^{5}$ is Hardy's generic probability of quantum entanglement [4] and which we used in previous measure theoretical analysis of the same problem [4-12]. 


\section{Further Remarks to the Sciama-Kibble Gravity}

To conclude this part of our discussion we may contemplate some obvious but important aspects of the Kibble scenario compared to the original Einstein scenario, i.e. the master plan for general relativity. Needless to mention Einstein's well known slight disdain for abtruse mathematics is a point which we paradoxically agree and disagree with him about. We move directly to the heart of the matter by noting the 20 independent Reimann tensor components of Einstein's gravity [22,23]

$$
\begin{aligned}
\mathrm{R}^{(4)} & =\mathrm{n}^{2}\left(\mathrm{n}^{2}-1\right) / 12 \\
& =(4)^{2}\left(4^{2}-1\right) / 12 \\
& =20
\end{aligned}
$$

One finds these 20 components by simply letting the constraints of Riemannian tensors vanish. The so obtained corresponding values in this case are 24 resulting from setting the torsional part of the Riemannian Cartan tensor equal zero while the other additional 36 result from setting nontorsional parts equal zero. Next we move to consider particle-like geometrical quantitites which because they are non-inertial may be considered particle-like quantum states. For Einstein scenario these are connected to the Christoffel symbols and there are 40 of them [14-19, 21-24]. In the Kibble scenario on the other hand there are our 24 which played a central role in our analysis and a second part, namely $40-24=16$ which are vital in any analysis of dark and ordinary energy density using the holographic boundary [25]. To be more specific it is easily shown that while [4-12]

$$
\begin{aligned}
\mathrm{E}(\mathrm{O}) & =\left(\frac{24}{528}\right)\left(\mathrm{mc}^{2}\right) \\
& =\mathrm{mc}^{2} / 22
\end{aligned}
$$

where 24 is $|\mathrm{SU}(5)|$ and 528 is $\mathrm{N}_{\mathrm{k}}^{(32)}$ when using the holographic boundary given by Lie symmetry group generators $|\operatorname{SL}(2,7)|=7\left(7^{2}-1\right)=336$ one finds

$$
\begin{aligned}
\mathrm{E}(\mathrm{O}) & =\left(\frac{16}{336+16}\right) \mathrm{mc}^{2} \\
& =\mathrm{mc}^{2} / 22
\end{aligned}
$$

exactly as should be. We recall here that 336 are also equal to the number of degrees of freedom of Klein's original modular curve $\Gamma(7)$. Similarly or analogously for dark energy we have [4-12]

$$
\begin{aligned}
E(D) & =\left(\frac{528-24}{588}\right) \mathrm{mc}^{2} \\
& =\mathrm{mc}^{2}(21 / 22)
\end{aligned}
$$

and using the holographic boundary [25]

$$
\begin{aligned}
E(D) & =\left(\frac{336}{336+16}\right) m c^{2} \\
& =m c^{2}(21 / 22) .
\end{aligned}
$$

Here $336+16=352$ are nothing but the super symmetric degrees of freedom of a massless graviton for $\mathrm{N}=8$ or the degrees of freedom of eight copies of an eleven dimensional pure gravity with 44 degrees of freedom, i.e. $8\left[\mathrm{D}^{(\mathrm{d})}=\mathrm{d}(\mathrm{d}\right.$ $-3) / 2]=8[11(11-3) / 2]=(8)(44)=352[22-24]$.

\section{Discussion and the importance of be- ing transfinitely fuzzy}

At this point a question must start pressing itself on any attentive reader or in fact the present Author himself, namely of why did conventional theories fail to uncover all the preceding surprisingly simple relations much earlier and why is it then only partially uncovered after considerable time and amount of research effort? We think the reason must be that all our conventional methods are based on basically non-fractal models and crisp non-fuzzy logic. It is then a Sisyphus task to try to find the fractal fine details of reality when we have started by washing them out. It only became with the incredible perseverance of superb theoretical physicists like Yang, Wilson, 't Hooft, Veltman and Witten that they could extract various vital information from incredible and for the normal scientist immensely messy and almost intractable algebraic manipulation and buffterfly effects of numerical computation with or without super computers [21]. It is simply our good fortune that we have the golden mean binary system at our disposal which reduces what could have been a mammoth computer program running for days to a simple half an hour of calculation using a modern small calculator $[21,33]$. Even more importantly our result is exact because we take the limit to infinity without taking the infinitesimal limit of Newton's calculus. In other words we have mathematical machinery which gives a transfinite final result by summing uncountably infinite series. This somewhat surprising result could be summarized in the following single but loaded anti L. Kronecker statement "God created the irrational transfinite golden mean numbers and all else is the work of man".

\section{Nonlocal Elasticity and Dark Energy}

In a recent paper [34] a most profound conclusion was made, namely the equivalence of the author's finite element discrete analysis [35,36] and A.C. Eringen's acclaimed theory of nonlocal elasticity [37]. The point is extremely important for quantum field theory and dark energy as well as to the theory of relativity [38] so that it is instructive to dwell on this subject a little.

Let us start from the total internal energy of any general elastic space [35-37]. Independent from the dimensionality of this space the total strain energy, i.e. the internal energy is the sum of (1) the bending energy, i.e. the curvature, (2) the stretching energy, i.e. the metric energy, (3) the energy of the shear forces and then (4) we must add the torsional energy. 
It is remarkable to note that Einstein's original theory of relativity took only one of these four different types of energy, namely the bending energy expressed in terms of the Riemann curvature. Even more ironic, when Cartan and Einstein realized the afore mentioned deficiency and introduced their telleparallel theory of relativity [14-16] they went to the other extreme and took de facto only the torsional energy and nothing else. On the other hand it seems that the only theory which accounts for everything is the theory of the brothers Cosserat [17,18]. Looking carefully at the situation one immediately realizes that only spacetime theories regard spacetime as truly similar to matter fields, i.e. granular could represent reality in an adequate manner [14,39]. It turned out that there are in principle a few theories which could account for this transfinite granular discreteness of spacetime, namely theories with extra dimensions, notably the five super string theories as well as $\mathrm{M}$ and $F$ theory $[12,23,32]$. In addition all theories admitting directly or indirectly lumping and discreteness of spacetime could also solve the basic problem with different degrees of accuracy. The most prominent among such theories which "pinch" space to a neural network is loop quantum gravity as well as Penrose twistor theory. What we have to avoid at all costs is to take the naïve limit to the continuum as done in any analysis relying mainly on Newton's differential calculus. It is this smoothing effect of classical calculus applied to space without extra dimensions which kills fundamental phenomena such as Hartle-Hawking fluctuation, Unruh temperature and Hardy's entanglement [9,10]. That way one could not understand why dark energy is localized at the edge of the infinite universe and where it comes from as well as why it has an anticlastic negative curvature producing negative gravity [39-45]. In recent papers we have shown that nonlocal elasticity is one way to account for the real material-like nature of spacetime and produce the same results and conclusions reported in the present work [39].

\section{Wider Philosophical Implications of the Theory and Conclusions}

Mountain climbers and W. Heisenberg was one of them in the metaphorical and literal meaning of the words [46] know that there are two fundamental attitudes towards this demanding sport-art. The first is to survey the mountain, have a global look at it, look how high and rugged it is, then identify the starting point and the approximate path to the summit. The second is just to concentrate on the first 100 meters and never look down or up too much or get distracted or scared by what still lies ahead. Needless to say, both attitudes are complimentary and under no circumstances exclusive. It is this inclusivity as opposed to exclusivity which we would like to emphasize here as the most important and distinctive feature of our theory as seen by an eagle which does not require the techniques nor the gear of a mountain climber.

On taking the eagle eye view of our theory, the first strange feature of the mountainscape would be that the summit and valleys are now flat and lie on the same contour lines with only a numerical index indicating the height.
Our space unites the ununited and what was separated "by man" is no more "asunder" Our space is open and closed at the same time. That elevates the simple concept which we take as self evident to the higher reality of topology, namely a clopen space. Similarly our space is infinite and yet finite. Again the obvious contradiction is resolved by the hyperbolic projection onto the plane "created" by "nature" via the work of Poincaré, Beltrami and Lobachevski in France, Italy and Russia (Kazan) respectively $[11,12,47]$. In our space what seems divergent may be readily resummed to give a finite answer via Borel techniques [41] as shown in " $t$ Hooft's analytical work and Wilson computer "simulation" [41]. The E-infinity spacetime is not fixed by a single dimension but by a host of various dimensions fitting together in a mathematical symphony. Formally the space has infinite dimensions yet because of its fundamental Cantorian hierarchal structure, it has a topological Menger Urysohn dimension $\mathrm{D}=4$ in addition to an expectation topological dimension $<\mathrm{n}>=4+\phi^{3}=4.23606799$ which happens to be equal to its Hausdorff dimension [47]. This is not all. It still has a scaling dimension equal to $4-\varepsilon$ where $\varepsilon$ is equal to twice Hardy's quantum entanglement as well as a spreading "spectral" dimension equal to 4.0199999 first discovered by R. Loll and J. Ambjørn and confirmed as distinct by L. Marek-Crnjac and the present author $[11,12,40,41]$. The reader must have noticed that we have descended from the global to the particulars. However this is only momentarily due to the complimentarity of the situation. To wrap the eagle eye view in a few mathematical terms may be a little hard to do because $n$ categories, E-infinity and Grothendieck K-theory [38-47] are too mathematical for most physicists including the present author. An exception in this regard may be the beautiful work of A. Connes on noncommutative geometry. In philosophical terms our space may be the geometrical topological incarnation of Hegel's dialectic and his coincidentia oppositorum [44]. At the end real experimental confirmation is what decides about what a potent scientific philosophy is. We show here beyond any reasonable doubt that our theoretical derivation of dark energy is in superb agreement with accurate cosmic measurements as well as the ultra symmetric logic of an all inclusive discrete nonlocal granular and physically real spacetime [12-18,39].

\section{References}

[1] E.V. Linder, Resource Letter: Dark energy and accelerating universe. arXiv: 0705.4102V1[astro-ph]28May 2007.

[2] S. Perlmutter and B. Schmidt, Measuring cosmology with supernova. arXiv: astro-ph/0303428V1 18 Mar 2003.

[3] E.V. Linder, Einstein's other gravity and the acceleration of the universe. arXiv: 1005.3039V2[astro-hy.co] 26 August 2010 .

[4] M.S. El Naschie, A resolution of cosmic dark energy via a quantum entanglement relativity theory. J. Quantum Info. Sci., Vol. 3(1), 2013, pp. 23-26. 
[5] M.S. El Naschie, Quantum gravity and dark energy via a new Planck scale. Fractal Spacetime \& Noncommutative Geometry in Quantum \& High Energy Phys., Vol. 3(2), 2013, pp. 106-119.

[6] M.S. El Naschie, Dark energy via a quantum field theory in curved spacetime. J. Mod. Phys. \& Appli., Vol. 1, 2014, pp. 1-7.

[7] M.S. El Naschie, From Yang-Mills photon in curved spacetime to dark energy density. J. Quantum Info. Sci., Vol. 3(4), 2013, pp. 121-126.

[8] L. Marek-Crnjac et al, Chaotic fractal tiling for the missing dark energy and Veneziano model. Appl. Math., Vol. 4(11B), 2013, pp. 22-29.

[9] M.S. El Naschie, A Rindler-KAM spacetime geometry and scaling the Planck scale solves quantum relativity and explains dark energy. Int. J. of Astronomy and Astrophysics, Vol. 3(4), 2013, pp. 483-493.

[10] M.S. El Naschie, Experimentally based theoretical arguments that Unruh's temperature, Hawking's vacuum fluctuation and Rindler's wedge are physically real. American J. Mod. Phys., Vol. 2(6), 2013, pp. 357-361.

[11] L. Marek-Crnjac and Ji-Huan He, An invitation to El Naschie's theory of Cantorian spacetime and dark energy. Int. J. of Astron. \& Astrophys., Vol. 3, 2013, pp. 464-471.

[12] L. Marek-Crnjac. and M.S. El Naschie, Quantum gravity and dark energy using fractal Planck scaling. J. Mod. Phys., Vol. 4(11A), 2013, pp. 31-38.

[13] T.W.B. Kibble, Lorentz invariance and the gravitational field. J. Math. Phys., Vol.2, 212 1961, pp. 212-221.

[14] F. Hehl, Space-Time as Generalized Cosserat Coninuum. In "Mechanics of Generalized Continua". Editor E. Kronev., Springer Verlag, Berlin 1968. pp. 347-349.

[15] A. Einstein, Auf die Riemann-Metrik und den Fern-Parallelismus gegründete einheitliche Feldtheorie. Mathematische Annalen, Vol. 102, 1930, pp. 685-697.

[16] E. Cartan, Notice historique sur la notion de parallelism absolu. Mathematische Annalen, Vol. 102, 1930, pp. 698-706.

[17] E. Cosserat, and F. Cosserat, Théorie des corps déformables. Hermann, Paris 1909.

[18] M. Blagojevic and M. Vasilic, Asymptotic symmetry and conserved quantities in the Poincaré gauge theory of gravity. Classical \& Quantum Gravity, Vol. 5, 1988, pp. 1241-1257.

[19] J. Burnett, Olga Chervova and Dmitri Vassiliev, Dirac equation as a special case of Cosserat elasticity. arXiv: 0812.3948V1[gr-qc]22 December 2008.

[20] M.S. El Naschie, Nash embedding of Witten's M-theory and the Hawking-Hartle quantum wave of dark energy. J. Mod. Phys., Vol. 4, 2013, pp. 1417-1428.

[21] M.S. El Naschie, Ji-Huan He, S. Nada, L. Marek-Crnjac and M.A. Helal, Golden mean computer for high energy physics, Fractal Spacetime and Noncommutative Geometry in High Energy Phys., Vol. 2(2), 2012, pp. 80-92.

[22] Ji-Huan He, Transfinite Physics. A collection of publications on E-infinity Cantorian spacetime theory. China Scientific \& Cultural Publishing. ISBN 988-9 8846-5-8 2005.
[23] M.J. Duff: M.J.: The World in Eleven Dimensions. Inst. of Phys. Publications, Bristol 1999.

[24] E. Witten, $2+1$ dimensional gravity as an exactly soluble system. Nucl. Phys. B. Vol. 311(1), 1988, pp. 46-78.

[25] D. Kutasov and N. Seiberg, Number of degrees of freedom, density of states and tachyons in string theory and CFT. Nucl. Phys. B, Vol. 358(3), 1991, pp. 600-618.

[26] M.S. El Naschie, On two new fuzzy Kähler manifolds, Klein modular space and 't Hooft holographic principles. Chaos, Solitons \& Fractals, Vol. 29(4), 2006, pp. 876-881.

[27] G. 't Hooft, A Confrontation With Infinity. In 'Frontiers of Fundamental Physics' 4. Editors B. Sidharth and M. Altaisky. Kluwer-Plenum, New York 2001, pp. 1-12.

[28] M.S. El Naschie, 't Hooft's dimensional regularization implies transfinite Heterotic string theory and dimensional transmutation. In 'Frontiers of Fundamental Physics' 4. Editors B. Sidharth and M. Altaisky. Kluwer-Plenum, New York 2001, pp. 81-86.

[29] K.G. Wilson, Critical phenomena in 3.99 dimensions. Physica, Vol. 73, 1974, pp. 119-128.

[30] K.G. Wilson, The renormalization group and the $\in$ expansion. Phs. Reports, Vol. 12(2), 1974, pp. 75-200.

[31] M.S. El Naschie, COBE satellite measurement, hyperspheres, super strings and the dimension of spacetime. Chaos, Solitons \& Fractals, Vol. 9(8), 1998, pp. 1445-1471

[32] M. Kaku, Introduction to Superstrings and M-Theory. Springer, New York 1999.

[33] M.S. El Naschie et al: On the need for fractal logic in high energy quantum physics. Int. J. Mod. Nonlinear Theory \& Application. Vol. 1(3), 2012, pp. 84-92.

[34] Challamel, N., Wang, C.M. and Elishakoff, I.: Discrete systems behave as nonlocal structural elements; bending buckling and vibration analysis. Euro. J. Mech. A/Solids, Vol. 44, 2014, pp. $125-135$.

[35] El Naschie, M.S.: Stress, Stability and Chaos in Structural Engineering: An Energy Approach. McGraw Hill Int. Edi tions Civil Eng. Series., London, Tokyo (1990).

[36] Wifi, A.S., El Naschie, M.S., Al Athel, S., Wu, C.W. and Obeid, K.: Coluterized stress and stability analysis of engi neering structures. King Abdulaziz City for Science and Tech Publishing, Saudi Arabia, No. 21. (96 pages with Arabic translation of summary).

[37] Eringen, A.C. and Edelen, D.G.: On nonlocal elasticity. Int. J. Eng. Sci., Vol. 10(3), 1972, pp. 233-248.

[38] El Naschie, M.S.: Cosmic dark energy density from classical mechanics and seemingly redundant Riemannian infitely many tensor components of Einstein's general relativity. World J. Mech. 2014. In press.

[39] El Naschie, M.S.: Pinched material Einstein spacetime pro duces accelerated cosmic expansion. Int. J. Astron. \& Astrophys. Vol. 4(1), 2014 pp. 80-90.

[40] El Naschie, M.S. and Helal, A.: Dark energy explained via the Hawking-Hartle quantum wave and the topology of cosmic crystallography. Int. J. Astronomy \& Astrophys., Vol. 3(3), 2013, pp. 318-343. 
[41] El Naschie, M.S.: Cosmic dark energy from 't Hooft's di mensional regularization and Witten's topological quantum field pure gravity. J. of Qant. Info. Sci., 2014. In press.

[42] El Naschie, M.S.: Why E is not equal to $\mathrm{mc}^{2}$. J. of Modern Phys. In press. (2014).

[43] El Naschie, M.S., Marek-Crnjac, L., Hela, M.A. and He, Ji-Huan: A topological Magueijo-Smolin varying speed of light theory, the accelerated cosmic expansion and the dark energy of pure gravity. Appl. Math. 2014. In Press.

[44] El Naschie, M.S.: The meta energy of dark energy. Open J. of Philosophy, (2014). In press.
[45] El Naschie, M.S.: Einstein's general relativity and pure grav ity in a Cosserat and de Sitter-Witten spacetime setting as the explanation of dark energy and cosmic accelerated ex pansion. Int. J. Astron. \& Astrophys. 2014. In press.

[46] Heisenberg, W.: Der Teil und das Ganze. Piper, Munich (1969).

[47] El Naschie, M.S.: A review of E-infinity and the mass spec trum of high energy particle physics. Chaos, Solitons \& Fractals, Vol. 19(1), 2004, pp. 209-236. 